\title{
Metarhizium baoshanense sp. nov., a New Entomopathogen Fungus from Southwestern China
}

\author{
Zihong Chen ${ }^{1}$, Ling $\mathrm{Xu}^{2}$, Xiaona Yang ${ }^{2}$, Yaguan Zhang ${ }^{3 *}$ and Yuming Yang ${ }^{4}$ \\ ${ }^{1}$ Institute of Biological Resources of Gaoligong Mountains, Baoshan University, \\ Baoshan 678000 Yunnan, China \\ ${ }^{2}$ College of Resources and Environment, Baoshan University, Baoshan 678000, \\ Yunnan, China \\ ${ }^{3}$ College of Chemistry and Environmental Science, Qujing Normal University, \\ Qujing 655011 Yunnan, China \\ ${ }^{4}$ Yunnan Province Key Laboratory of Forest Plant Cultivation and Utilization, \\ Yunnan Academy of Forestry, Kunming 650201 Yunnan, China
}
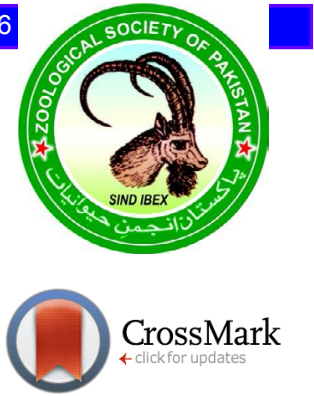

\begin{abstract}
A B S T R A C T
Metarhizium is an entomopathogenic fungal genus relevant in biological protection. A new species of the genus, collected from Taibao mountain, Baoshan City, Yunnan Province, southwestern China, was described here as Metarhizium baoshanense. It was proposed and determined based on morphological characters combined with a multigene phylogenetics analysis involving 5.8S-ITS, nrSSU, nrLSU, EF-1 $\alpha$, RPB1 and RPB2. In multilocus phylogeny, M. baoshanense was grouped as a sister clade to Metarhizium majus, Metarhizium guizhouense, Metarhizium lepidiotae and Metarhizium indigoticum, but conclusively separated from these allied species with high support value. Morphological character differentiated $M$. baoshanense from its relatives on that its conidial size was obviously smaller than $M$. majus and $M$. indigoticum, a little shorter than M. guizhouense and thicker than M. lepidiotae. Still the new species was characterized by that its colony colour became slightly shallow with culture time on PDA plates at $25^{\circ} \mathrm{C}$, being grayish green at $12 \mathrm{~d}$ and turning gray black at $30 \mathrm{~d}$ of cultivation.
\end{abstract}

\section{INTRODUCTION}

$M$ etarhizium is one of the ubiquitous anamorphic genera of entomopathogenic fungi (Driver et al., 2000). Entomophthoro anisopliae Metsch was described from Russia as a pathogen of wheat cockchafer (Anisolia austriaca Hbs.) and turned to be a type species of a new genus Metarhizium (Metsch.) Sorokin. Teleomorph of Metarhizium was firstly confirmed as Cordyceps s. 1. by Liang et al. (1991) and then assigned to a new genus Metacordyceps by Sung et al. (2007).

A high number of species and varieties of Metarhizium had been discovered in recent decades and their convergent morphologies hampered the taxonomic studies. Molecular evidence enabled great resolution of fungi taxonomy than allowed by morphology alone, and mutigene phylogenetic approaches particularly urged/forced the clarification of the genetic relationship at species rank (Kepler et al., 2012; Nonaka et al., 2013). Based on 4-gene (EF-1 $\alpha, \mathrm{RPB} 1$,

\footnotetext{
* Corresponding author: zhangyaguan2013@163.com 0030-9923/2018/0005-1739 \$ 9.00/0

Copyright 2018 Zoological Society of Pakistan
}

RPB2 and $\beta$-tubulin) dataset, Bischoff et al. (2009) revealed that the Metarhizium anisopliae complex included 9 species. Based on 5-gene (nrSSU, nrLSU, EF-1 $\alpha$, RPB1 and RPB2) dataset, Kepler et al. (2012) identified that Metacordyceps contained 14 species and 1 varietas, and then Kepler et al. (2014) revised the boundaries of Metarhizium as including the majority of species recognized in Metacordyceps, green-spored species in Nomuraea, Chamaeleomyces and several species formerly in Paecilomyces.

During a survey of entomogenous fungi at Taibao mountain in Baoshan City, Yunnan Province, China in 2015, a new Metarhizium species, M. baoshanense was discovered. The objective of this study was to confirm its taxonomy position based on morphological characteristics and 6-locus phylogenetic analysis. The anamorph of the related species, $M$. indigoticum (Kobayasi \& Shimizu) Kepler, S.A. Rehner \& Humber, was firstly described.

\section{MATERIALS AND METHODS}

Fungal isolation and morphological evaluation Soil specimens were collected from plant rhizosphere 
at mid-montane of humid evergreen broad-leaved forest in Taibao Mountain, Baoshan city, Yunnan Province in China. Fungi were isolated from soil by insect baiting method according to Keyser et al. (2015). Soil samples $(\sim 100 \mathrm{~mL})$ were moistened to slightly damp in sterile glass canned bottles $(150 \mathrm{~mL})$. Ten healthy $6-7$ th instar Tenebrio molitor L. larvae were cultured in each bottle and checked every two days. Dead insects were transferred to a sterile petri dish with moist filter paper and maintained at $25^{\circ} \mathrm{C}$ for about $10 \mathrm{~d}$. Metarhizium conidia were transferred to PDA plates and cultured at $25^{\circ} \mathrm{C}$. Pure (axenic) strains were indentified morphologically and evaluated microscopically under a motic BA410 microscope. Morphological characteristics of the new species were compared with its related species among $M$. anisopliae complex. Anamorphic strains of the related species, $M$. indigoticum, were isolated from Gaoligong mountains and morphologically described.

\section{DNA extraction and PCR}

Genomic DNA was extracted from mycelia and conidia with the Plant Genomic DNA Purification Kit (Qiagen) following the manufacturer instructions. The partial sequences of 6 nuclear loci, including 5.8S-ITS, nrSSU, nrLSU, EF-1 $\alpha$, RPB1 and RPB2, were amplified. Primer pairs of the 6-locus were referred to Chen et al. (2013). PCR reactions were carried out in $20 \mu \mathrm{L}$ reaction mixture containing $10 \mu \mathrm{L} 2 \times$ EasyTaq PCR Supermix (TransGen Biotech, Beijing, China), $1 \mu \mathrm{L}$ of each primer $(10 \mu \mathrm{M}), 1 \mu \mathrm{L}$ of template DNA (1-2 ng) and $7 \mu \mathrm{L}$ sterile water. PCR programs of ITS-5.8S, nrSSU and nrLSU were referred to Chen et al. (2013) and that of EF-1 $\alpha, \mathrm{RPB} 1$ and
RPB2 were referred to Bischoff et al. (2006).

\section{Cloning and sequencing}

PCR products were purified with Gel Purification Kit (Bioteke, Beijing, China) and the fragments were cloned with TaKaRa PMD ${ }^{\mathrm{TM}} 18-\mathrm{T}$ vector system (TaKaRa Bio, Dalian, China). DNA sequencing was performed at SinoGenoMax Co. Ltd., and the resulting datasets were submitted to GenBank.

\section{Phylogenetic analysis}

DNA sequences of 6-locus included 34 species, $M$. baoshanense, all dataset (30 species) in Metarhizium used by Kepler et al. (2014), Metarhizium martialis (Speg.) Kepler, G.H. Sung \& Spatafora used by Kepler et al. (2012), Metarhizium shibinensis T.C. Wen, J.C. Kang $\&$ K.D. Hyde used by Wen et al. (2015) and Beauveria bassiana (Bals.) Vuill. as the outgroup. All sequences were retrieved from the GenBank with accession number detailed in Table I.

The combined dataset of 6-locus were aligned with Clustal X2.0 (Larkin et al., 2007). Ambiguous regions in both sides were excluded and gaps were treated as missing data in the subsequent phylogenetic analyses. Phylogenic consensus tree was analyzed with the program MEGA6 (Tamura et al., 2013). Maximum Likelihood (ML) estimation was performed with 1000 replicates. T92+G+I (Tamura 3-parameter + Gamma distribution $(+\mathrm{G})$ with invariant sites $(+\mathrm{I})$ nucleotide substitution model was taken as suggested by looking for a best-fit substitution model (ML). Clades supported with ML values of $\geq 70 \%$ were considered significantly supported by the data.

Table I.- Taxon information and GenBank accession numbers of the sequences used for phylogenetic analysis.

\begin{tabular}{|c|c|c|c|c|c|c|c|}
\hline \multirow[t]{2}{*}{ Species } & \multirow[t]{2}{*}{ Strain code } & \multicolumn{6}{|c|}{ GenBank accession number } \\
\hline & & nrSSU & nrLSU & EF-1a & RPB1 & RPB2 & 5.8S-ITS \\
\hline Metarhizium acridum & ARSEF 324 & Data missing & Data missing & EU248844.1 & EU248896.1 & EU248924.1 & HМ055449.1 \\
\hline M. acridum & ARSEF 7486 & Data missing & Data missing & EU248845.1 & EU248897.1 & EU248925.1 & NR132019.1 \\
\hline M. album & ARSEF 2082 & DQ518775.1 & DQ522560.1 & DQ522352.1 & DQ522398.1 & DQ522452.1 & Data missing \\
\hline M. album & ARSEF 2179 & Data missing & Data missing & KJ398807.1 & KJ398618.1 & Data missing & HM055452.1 \\
\hline M. anisopliae & ARSEF 7450 & Data missing & Data missing & EU248852.1 & EU248904.1 & EU248932.1 & HQ331464.1 \\
\hline M. anisopliae & ARSEF 7487 & Data missing & Data missing & DQ463996.2 & DQ468355.1 & DQ468370.1 & NR132017.1 \\
\hline M. baoshanense & ССТСС М 2016589 & KY264177 & KY264174 & KY264169 & KY264180 & KY264183 & KY264172 \\
\hline M. baoshanense & BUM 63.4 & KY264178 & KY264175 & KY264170 & KY264181 & KY264184 & KY264173 \\
\hline M. baoshanense & BUM 1700 & KY264179 & KY264176 & KY264171 & KY264182 & KY264185 & Data missing \\
\hline M. brasiliense & ARSEF 2948 & Data missing & Data missing & KJ398809.1 & KJ398620.1 & Data missing & Data missing \\
\hline M. brunneum & ARSEF 2107 & Data missing & Data missing & EU248855.1 & EU248907.1 & EU248935.1 & NR132023.1 \\
\hline M. brunneum & ARSEF 4179 & Data missing & Data missing & EU248854.1 & EU248906.1 & EU248934.1 & HQ331451.1 \\
\hline M. carneum & CBS 239.32 & EF468843.1 & EF468988.1 & EF468789.1 & EF468894.1 & EF468938.1 & NR131993.1 \\
\hline M. cylindrosporum & ARSEF 6926 & Data missing & Data missing & KJ398814.1 & KJ398625.1 & Data missing & AF368270.1 \\
\hline
\end{tabular}




\begin{tabular}{|c|c|c|c|c|c|c|c|}
\hline \multirow[t]{2}{*}{ Species } & \multirow[t]{2}{*}{ Strain code } & \multicolumn{6}{|c|}{ GenBank accession number } \\
\hline & & nrSSU & nrLSU & EF-1a & RPB1 & RPB2 & 5.8S-ITS \\
\hline Metarhizium flavoviride & ARSEF 2133 & Data missing & Data missing & DQ463999.1 & DQ468358.1 & DQ468373.1 & Data missing \\
\hline M. flavoviride & ARSEF 2025 & Data missing & AF138269.1 & KJ398804.1 & KJ398614.1 & DQ468374.1 & AF138269.1 \\
\hline M. frigidum & ARSEF 7445 & Data missing & Data missing & KJ398818.1 & KJ398628.1 & Data missing & Data missing \\
\hline M. frigidum & ARSEF 4124 & Data missing & Data missing & DQ464002.1 & DQ468361.1 & DQ468376.1 & NR132012.1 \\
\hline M. globosum & ARSEF 2596 & Data missing & Data missing & EU248846.1 & EU248898.1 & EU248926.1 & NR132020.1 \\
\hline M. granulomatis & UAMH 11176 & HM635078.1 & Data missing & KJ398782.1 & KJ398593.1 & Data missing & HM195306.1 \\
\hline M. granulomatis & UAMH 11028 & HM195304.1 & HM635076.1 & KJ398781.1 & Data missing & Data missing & NR132013.1 \\
\hline M. guizhouense & CBS 258.90 & Data missing & Data missing & EU248862.1 & EU248914.1 & EU248942.1 & HQ331448.1 \\
\hline M. guizhouense & ARSEF 6238 & Data missing & Data missing & EU248857.1 & EU248909.1 & EU248937.1 & HQ331447.1 \\
\hline M. indigoticum & TNS-F 18553 & JF415968.1 & JF415952.1 & JF416010.1 & JN049886.1 & JF415992.1 & JN049874.1 \\
\hline M. indigoticum & TNS-F 18554 & JF415952.1 & JF415969.1 & JF416011.1 & JN049887.1 & JF415993.1 & JN049875.1 \\
\hline M. indigoticum & BUM 1512.8 & KY264192 & KY264190 & KY264186 & KY264194 & KY264196 & KY264188 \\
\hline M. indigoticum & BUM 2600 & KY264193 & KY264191 & KY264187 & KY264195 & KY264197 & KY264189 \\
\hline M. khaoyaiense & BCC 12687 & JF415971.1 & Data missing & KJ398796.1 & JN049889.1 & Data missing & Data missing \\
\hline M. khaoyaiense & BCC 14290 & JF415970.1 & Data missing & KJ398797.1 & JN049888.1 & Data missing & JN049869.1 \\
\hline M. koreanum & ARSEF 2039 & Data missing & Data missing & KJ398806.1 & KJ398616.1 & Data missing & Data missing \\
\hline M. koreanum & ARSEF 2038 & Data missing & Data missing & KJ398805.1 & KJ398615.1 & Data missing & Data missing \\
\hline M. kusanagiensis & TNS-F 18494 & JF415972.1 & JF415954.1 & JF416014.1 & JN049890.1 & Data missing & JN049873.1 \\
\hline M. lepidiotae & ARSEF 7412 & Data missing & Data missing & EU248864.1 & EU248916.1 & EU248944.1 & HQ331455.1 \\
\hline M. majus & ARSEF 1946 & Data missing & Data missing & EU248867.1 & EU248919.1 & EU248947.1 & HM055450.1 \\
\hline M. majus & ARSEF 1914 & Data missing & Data missing & KJ398801.1 & KJ398610.1 & Data missing & HQ331445.1 \\
\hline M. marquandii & CBS 182.27 & EF468845.1 & EF468990.1 & EF468793.1 & EF468899.1 & EF468942.1 & NR131994.1 \\
\hline M. martialis & HMAS 197472 & JF415956.1 & JF415975.1 & JF416016.1 & JN049892.1 & JF415995.1 & JN049881.1 \\
\hline M. minus & ARSEF 1764 & AF280635.1 & AF280632.1 & DQ464006.1 & KJ398609.1 & DQ468380.1 & HM055453.1 \\
\hline M. minus & ARSEF 2037 & AF339531.1 & AF339580.1 & DQ464007.1 & DQ468366.1 & DQ468381.1 & AF138271.1 \\
\hline M. novozealandicum & ARSEF 4661 & Data missing & Data missing & KJ398811.1 & KJ398622.1 & Data missing & Data missing \\
\hline M. novozealandicum & ARSEF 4674 & Data missing & Data missing & KJ398812.1 & KJ398623.1 & Data missing & Data missing \\
\hline M. owariense & NBRC 33258 & HQ165730.1 & HQ165669.1 & HQ165689.1 & HQ1665747.1 & Data missing & HQ165712.1 \\
\hline M. pemphigi & ARSEF 7491 & Data missing & Data missing & KJ398819.1 & KJ398629.1 & DQ468379.1 & Data missing \\
\hline M. pemphigi & ARSEF 6569 & Data missing & Data missing & KJ398813.1 & KJ398624.1 & DQ468378.1 & Data missing \\
\hline M. pinghaense & CBS 257.90 & Data missing & Data missing & EU248850.1 & EU248902.1 & EU248930.1 & NR077205.1 \\
\hline M. pinghaense & ARSEF 4342 & Data missing & Data missing & EU248851.1 & EU248903.1 & EU248931.1 & HQ331454.1 \\
\hline M. pseudoatrovirens & TNSF 16380 & JF415977.1 & Data missing & Data missing & JN049893.1 & JF415997.1 & JN049870.1 \\
\hline M. rileyi & ARSEF 1972 & Data missing & Data missing & KJ398803.1 & KJ398613.1 & Data missing & Data missing \\
\hline M. rileyi & CBS 806.71 & Data missing & AY526491.2 & EF468787.1 & EF468893.1 & EF468937.1 & NR119513.1 \\
\hline M. robertsii & ARSEF 727 & Data missing & Data missing & DQ463994.1 & DQ468353.1 & DQ468368.1 & HQ331453.1 \\
\hline M. viride & ARSEF 2456 & Data missing & Data missing & KJ398808.1 & KJ398619.1 & Data missing & EU553291.1 \\
\hline M. viride & CBS 659.71 & HQ165735.1 & HQ165673.1 & HQ165692.1 & Data missing & HQ165652.1 & HQ165714.1 \\
\hline M. viridulum & ARSEF 6927 & Data missing & Data missing & KJ398815.1 & KJ398626.1 & Data missing & Data missing \\
\hline M. yongmunense & EFCC 2135 & Data missing & EF468979 & EF468834 & EF468769 & EF468877 & Data missing \\
\hline M. yongmunense & EFCC 2131 & EF468833.1 & EF468977.1 & EF468770.1 & EF468876.1 & Data missing & JN049856.1 \\
\hline M. shibinensis & GZUHSB 13050311 & KR153588.1 & Data missing & KR153589.1 & KR153590.1 & Data missing & KR153585.1 \\
\hline Beauveria bassiana & ARSEF 7518 & HQ880975.1 & HQ880975.1 & HQ880975.1 & HQ880834.1 & HQ880834.1 & HQ880762.1 \\
\hline
\end{tabular}




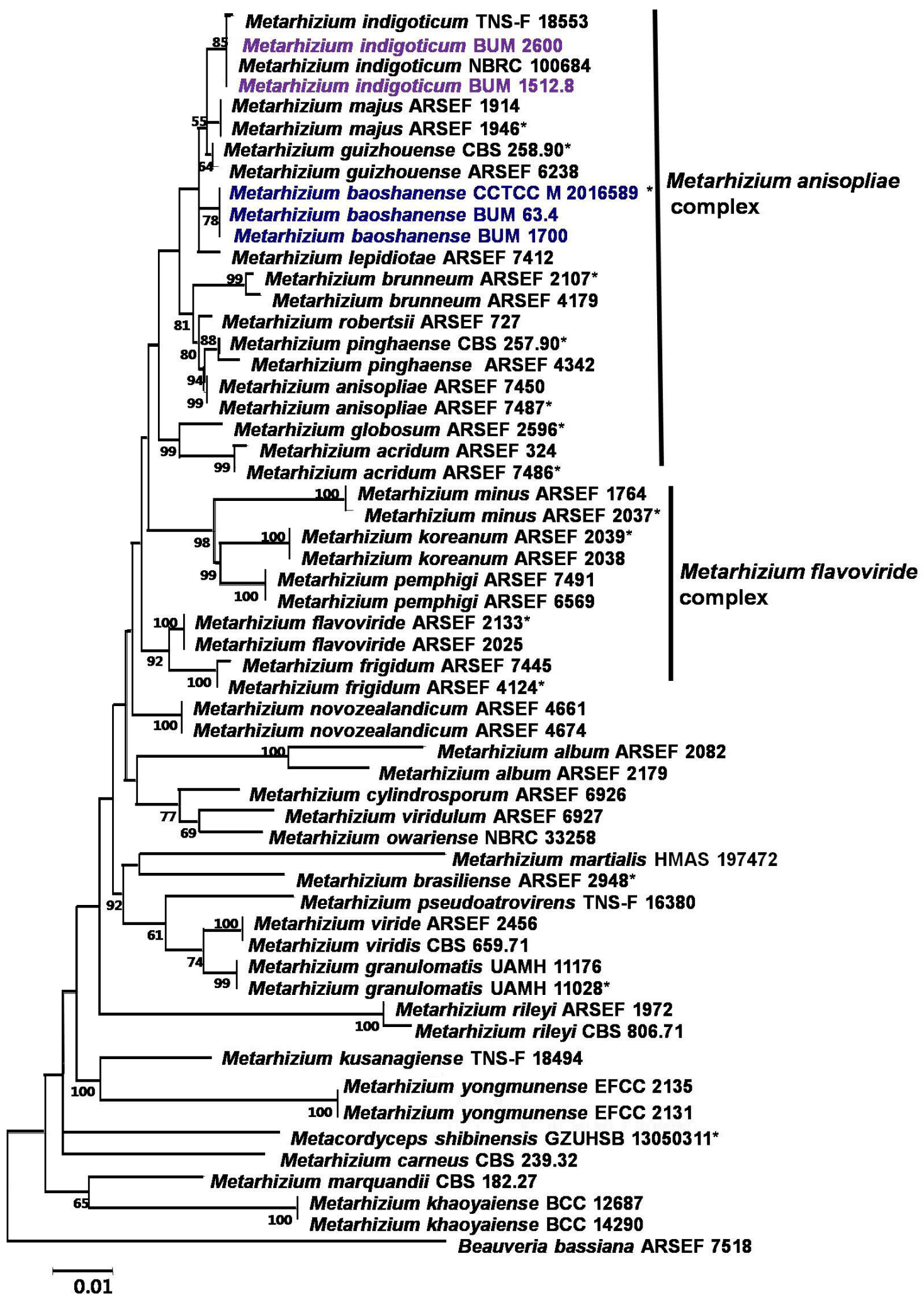

Fig. 1. Phylogenetic tree of Metarhizium based on ML analysis of 6-locus (5.8S-ITS, nrSSU, nrLSU, EF-1 $\alpha$, RPB1 and RPB2) dataset. *Denotes ex-type strains. 


\section{RESULTS}

Phylogenetic analyses

BLAST analysis revealed that 6 genes of $M$. baoshanense respectively shared sequences identities with different species of Metarhizium and Metacordyceps, ITS 98\% with M. anisopliae M3419 (FJ609313.1), EF-1 $\alpha$ 99\% with M. majus ARSEF 7505 (EU248870.1), RPB1 99\% with M. indigoticum NBRC 100684 (KJ398595.1), RPB2 99\% with Metacordyceps brittlebankisoides (KC429019.1), nrLSU and nrSSU 98\% with Metacordyceps indigotica TNS-F 18554 (JF415953.1). It was hard to determine the species position with a single gene. Further multilocus analysis was employed to confirm the species taxonomic status.

The combined alignment of 6-locus dataset of Metarhizium comprised 5049 base pairs (5.8S-ITS: 717 bp; nrSSU: 1018 bp; nrLSU: 846 bp; EF-1 $\alpha$ : 917 bp; RPB1: $713 \mathrm{bp}$; RPB2: 838 bp). Phylogenetic tree of combined 6locus for 34 species revealed similar affiliation inferred from 5 nuclear loci by Kepler et al. (2014) (Fig. 1). M. baoshanense was clustered in $M$. anisopliae complex which contained 11 individuals. The 3 strains (CCTCC M 2016589, BUM 63.4 and BUM 1700) of M. baoshanense were clustered in the same clade with M. majus, $M$. indigoticum, M. guizhouense Q.T. Chen \& H.L. Guo and M. lepidiotae (Driver \& Milner) J.F. Bisch., Rehner \& Humber, but formed a distinct clade and was clearly separated from the allied species with high support value.

The position of the strains BUM 2600 and BUM 1512.8 isolated from Gaoligong mountains was also confirmed. Their 6-locus dataset was clustered in the same clade with the reported sequences (TNS-F18553 and NBRC 100684) of $M$. indigoticum, illustrating that they were the anamorph stage of $M$. indigoticum and their morphological data in this study was credible.

Morphological comparison of M. Baoshanense and its related species

M. baoshanense was obviously distinguished from $M$. majus and $M$. indigoticum for the conidial feature, $M$. majus possessing the biggest conidia $(8.5-13.0 \times 3.0-5.0$ $\mu \mathrm{m})$ in $M$. anisopliae complex and $M$. indigoticum (8.2$12.3 \times 2.6-4.2 \mu \mathrm{m})$ being next to it, much greater than $M$. baoshanense $(6.7-8.5 \times 2.6-3.3 \mu \mathrm{m})$ (Table II). The conidia of M. baoshanense were close to M. lepidiotae (6.0$7.5 \times 2.0-3.0 \mu \mathrm{m})$ for the length and a little shorter than $M$. guizhouense $(7.0-10.0 \times 2.0-3.0 \mu \mathrm{m})$, while being a little thicker than M. guizhouense and M. lepidiotae (Table II; Fig. 2I-L).

Table II.- Morphological characterscomparison among Metarhiziumanisopliae complex.

\begin{tabular}{|c|c|c|c|c|c|}
\hline Species & Strain & Geography & Conidia $(\mu \mathrm{m})$ & Phialides $(\mu \mathrm{m})$ & Reference \\
\hline Metarhizium majus & ARSEF 1914 & Philippines & $10.5-13.0 \times 2.5-4.0$ & $10.0-18.0 \times 2.5-3.5$ & Bischoof et al. (2009) \\
\hline M. majus & ARSEF 7505 & Australia & $8.5-13.0 \times 3.0-5.0$ & $9.0-18.5 \times 2.5-4.5$ & Bischoof et al. (2009) \\
\hline M. indigoticum & BUM 1512.8 & China & $\begin{array}{c}8.2-12.3 \times 2.6-4.2 \\
(9.7 \pm 1.0 \times 3.1 \pm 0.6)\end{array}$ & $\begin{array}{c}8.5-20.5 \times 1.7-3.5 \\
(13.7 \pm 3.7 \times 2.5 \pm 0.6)\end{array}$ & This study \\
\hline M. guizhouense & ARSEF 4321 & Australia & $5.5-8.0 \times 2.5-3.5$ & $8.0-12.0 \times 2.5-3.5$ & Bischoof et al. (2009) \\
\hline M. guizhouense & CBS 258.90 & China & $7.0-10.0 \times 2.0-3.0$ & $7.0-12.0 \times 2.0-3.0$ & Bischoof et al. (2009) \\
\hline M. guizhouense & ARSEF 4321 & Australia & $5.5-8.0 \times 2.5-3.5$ & $8.0-12.0 \times 2.5-3.5$ & Bischoof et al. (2009) \\
\hline M. baoshanense & ССТCC M 2016589 & China & $\begin{array}{c}6.7-8.5 \times 2.6-3.3 \\
(7.4 \pm 0.5 \times 2.9 \pm 0.3)\end{array}$ & $\begin{array}{c}8.2-19.2 \times 2.1-3.5 \\
(11.9 \pm 3.3 \times 2.5 \pm 0.4)\end{array}$ & This study \\
\hline M. lepidiotae & ARSEF 4154 & Australia & $6.0-7.5 \times 2.0-3.0$ & $6.5-12.0 \times 2.0-3.0$ & Bischoof et al. (2009) \\
\hline M. lepidiotae & ARSEF 7488 & Australia & $5.0-7.0 \times 3.0-4.0$ & $8.0-12.0 \times 2.5-3.5$ & Bischoof et al. (2009) \\
\hline M. pingshaense & CBS 257.90 & China & $6.0-8.0 \times 2.5-3.5$ & $7.0-17.0 \times 2.5-3.5$ & Bischoof et al. (2009) \\
\hline M. pingshaense & ARSEF 4342 & Solomon islands & $4.5-7.0 \times 2.0-3.0$ & $7.0-13.0 \times 2.0-3.0$ & Bischoof et al. (2009) \\
\hline M. robertsii & ARSEF 7501 & Australia & $5.0-7.5 \times 2.0-3.0$ & $6.0-12.0 \times 2.0-3.0$ & Bischoof et al. (2009) \\
\hline M. robertsii & ARSEF 727 & Brazil & $5.0-7.0 \times 2.0-3.5$ & $7.0-14.5 \times 2.5-3.5$ & Bischoof et al. (2009) \\
\hline M. anisopliae & ARSEF 7487 & Eritrea & $5.0-7.0 \times 2.0-3.5$ & $8.0-11.5 \times 2.0-3.0$ & Bischoof et al. (2009) \\
\hline M. brunneum & ARSEF 4179 & Australia & $5.5-8.0 \times 2.0-3.0$ & $6.0-13.0 \times 2.0-3.0$ & Bischoof et al. (2009) \\
\hline M. brunneum & ARSEF 2107 & USA & $4.5-7.5 \times 2.0-3.0$ & $9.5-18.0 \times 2.0-5.0$ & Bischoof et al. (2009) \\
\hline M. brunneum & ARSEF 4152 & Australia & $5.0-7.0 \times 2.5-3.5$ & $7.5-13.5 \times 2.5-4.0$ & Bischoof et al. (2009) \\
\hline M. acridum & ARSEF 5748 & Mexico & $4.0-5.0 \times 2.5-3.5$ & $6.0-13.0 \times 2.0-3.5$ & Bischoof et al. (2009) \\
\hline M. acridum & ARSEF 7486 & Niger & $4.0-5.5 \times 3.0-4.0$ & $4.5-12.5 \times 2.5-4.5$ & Bischoof et al. (2009) \\
\hline M. globosum & ARSEF 2596 & India & $4.0-5.0 \times 4.0-5.0$ & $5.0-12.0 \times 3.0-4.0$ & Bischoof et al. (2009) \\
\hline
\end{tabular}



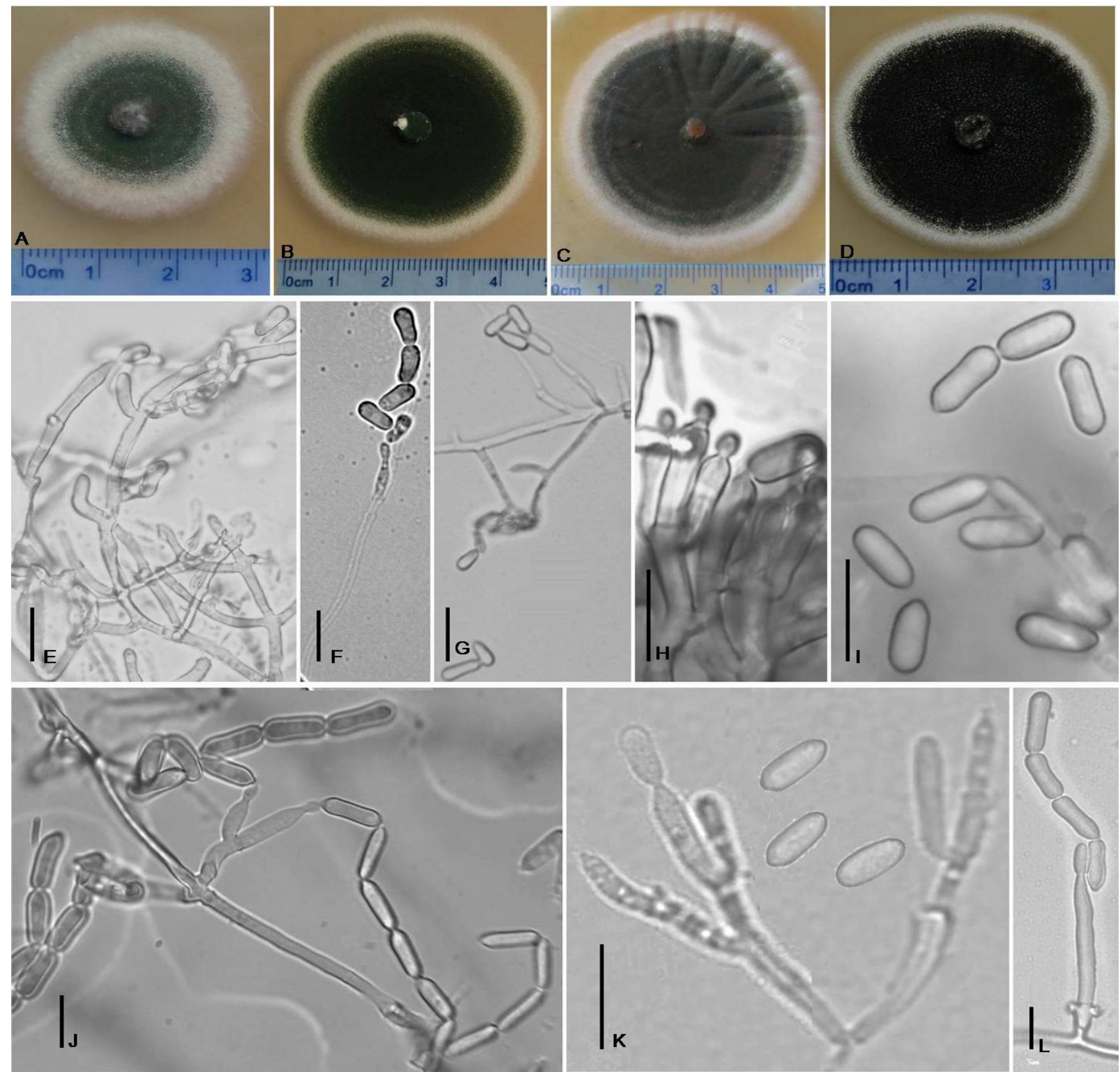

Fig. 2. Morphology of Metarhizium baoshanense and its close related species. A, colony of Metarhizium baoshanense cultured $12 \mathrm{~d}$; B, colony of Metarhizium lepidiotae cultured $12 \mathrm{~d}$; C, colony of Metarhizium guizhouense cultured $12 \mathrm{~d}$; D, colony of Metarhizium indigoticum cultured $12 \mathrm{~d}$; E, phialides formation of Metarhizium baoshanense; F and G, phialide with conidia in chain of Metarhizium baoshanense; $\mathrm{H}$, phialide with budding conidium of Metarhizium baoshanense; I, conidia in chain and separated of Metarhizium baoshanens; J, phialides with conidia in chain of Metarhizium guizhouense; K, conidia and phialides of Metarhizium lepidiotae; L, conidia and phialides of Metarhizium indigoticum. Bars, E-L $10 \mu \mathrm{m}$.

The culture character was further conducted among M. baoshanense, M. lepidiotae, M. guizhouense and $M$. indigoticum (Fig. 2 A-D). The conidia layers of all the four species were white in early day, while showed apparent discrepancy with culture time. After $12 \mathrm{~d}$ of cultivation on
PDA plates at $25^{\circ} \mathrm{C}, M$. baoshanense was grayish green (Fig. 2A), M. guizhouense was blackish green (Fig. 2B), M. lepidiotae was light black (Fig. 2C) and M. indigoticum was dark black (Fig. 2D). After one month of cultivation, $M$. baoshanense and $M$. lepidiotae were gray black, $M$. 
guizhouense was dark green, and M. indigoticum was black.

\section{Taxonomy \\ MycoBank number \\ MB 819373.}

\section{Species diagnosis}

The new species is clustered with $M$. guizhouense, $M$. lepidiotae, M. majus and M. indigoticum in the same clade in the multiple gene (5.8S-ITS, nrSSU, nrLSU, EF-1 $\alpha$, RPB1 and RPB2) sequence analyses, but the conidial size of $M$. baoshanense was obviously smaller than M. majus and $M$. indigoticum, a little shorter than M. guizhouense and thicker than $M$. lepidiotae.

\section{Species description}

Colonies on PDA medium being $61 \mathrm{~mm}$ in diameter after $16 \mathrm{~d}$ at $25^{\circ} \mathrm{C}$, white at first, turning grayish green after $16 \mathrm{~d}$ of cultivation. Hyphae hyaline, septate, branched, smooth-walled, $2.0-3.2(\bar{x}=2.5 \pm 0.6) \mu \mathrm{m}$ wide. Conidiophores solitary or branched. Phialides cylindrical, $8.2-19.2 \times 2.1-3.5(\bar{x}=11.9 \pm 3.3 \times 2.5 \pm 0.4) \mu \mathrm{m}$. Conidia forming columns in culture and hyaline, aseptate, smooth, long oval or cylindrical, grayish green en masse, $6.7-8.5 \times$ 2.6-3.3 $(\bar{x}=7.4 \pm 0.5 \times 2.9 \pm 0.3) \mu \mathrm{m}$.

Type: China. Yunnan Province: Taibao mountain, alt. 1740 m, 3 May 2015, Zihong Chen (Holotype, CCTCC M 2016589; ex-type culture, BUM 63.4)

Sexual state: Unknown.

Host: Unknown.

Habitat and distribution: The soil at mid-montane of humid evergreen broad-leaved forest in Taibao mountain, Baoshan city, Yunnan Province, China.

\section{DISCUSSION}

Most species in Metarhizium are useful biological control agents and often act as regulators of insect populations (Kepler et al., 2014; Pattemore et al., 2014; Shoukat et al., 2018). Further understanding their diversity can provide new genetic resource for development the entomopathogen as a biopesticide. In this study, $M$. baoshanense was determined to be a new species in Metarhizium based on morphological study and molecular evidence.

Muti-locus phylogeny is routinely used to delimit species of Ascomycota (Wang et al., 2015). Six loci including 5.8S-ITS, nrSSU, nrLSU, EF-1 $\alpha$, RPB1 and RPB2 were performed analysis in this study for their wide usage in phylogenetic reconstructions of fungi (Kepler et al., 2012; Sanjuan et al., 2014; Wen et al., 2015). Multi- genes analyses involved almost all currently recognized species of Metarhizium and the resulted affiliation was consistent with the works of Kepler et al. (2014), being able to provide strong evidence for the taxonomic status of M. baoshanense. The 2 strains BUM 2600 and BUM 1512.8 were placed as $M$. indigoticum and the 3 strains of $M$. baoshanense were resolved as a novel clade in M. anisopliae complex, clearly separated from its allied species, M. majus, M. guizhouense, M. lepidiotae and $M$. indigoticum.

Cordyceps indigotica Kobayasi \& Shimiz was firstly reported by Kobayasi and Shimizu (1978) and determined to be a member of Metacordyceps by Kepler et al. (2012). However, so far the asexual morphological description of $M$. indigoticum had been still missing. To better clarify the relationship of $M$. baoshanense and its related species, 2 strains from Gaoligong Mountains were confirmed as $M$. indigoticum by molecular analysis and morphologically compared with M. baoshanense (Table II). The conidial size of $M$. baoshanense was obviously smaller than M. majus and $M$. indigoticum, a little shorter than M. guizhouense, and a little thicker than Metarhizium lepidiotae. The colony color could well clarify the interspecific relationship, that of $M$. baoshanense being grayish green, obviously shallow than $M$. lepidiotae, M. guizhouense and M. indigoticum after $12 \mathrm{~d}$ of cultivation on PDA plates at $25^{\circ} \mathrm{C}$.

\section{CONCLUSION}

In this study, a new species of Metarhizium, $M$. baoshanense was determined based on morphological characters and 6-locus (5.8S-ITS, nrSSU, nrLSU, EF-1 $\alpha$, RPB1 and RPB2) molecular data. M. baoshanense was obviously separated from its sister species, M. majus, M. guizhouense, M. lepidiotae and $M$. indigoticum in phylogenetic tree and was also clearly morphologically distinguished from its relatives.

\section{ACKNOWLEDGEMENT}

The authors wish to thank Dr. Yong-Dong Dai (Institute of Herbal Biotic Resources, Yunnan University, Yunnan, China) for his kindly help to analyse the multigene sequences. This work was funded by the National Natural Science Area Fund Projects of China (31460153), Key Project of Universities Joint Foundation in Yunnan province (2017FH001-126), Surface Project of Universities Joint Foundation OF Yunnan province (2017FH001-029).

\section{Statement of conflict of interest}

The authors declare no conflicts of interest. All the 
experiments undertaken in this study comply with the current laws of the country where they were performed.

\section{REFERENCES}

Bischoff, J.F., Rehner, S.A. and Humber, R.A., 2006. Metarhizium frigidum spp. Nov.: a cryptic species of $M$. anisopliae and a member of the $M$. flavoviride complex. Mycologia, 98: 737-745. https://doi. org/10.3852/mycologia.98.5.737

Bischoff, J.F., Rehner, S.A. and Humber, R.A., 2009. A multilocus phylogeny of the Metarhizium anisopliae lineage. Mycologia, 101: 512-530. https://doi.org/10.3852/07-202

Chen, Z.H., Dai, Y.D., Yu, H., Yang, K., Yang, Z.L., Yuan, F. and Zeng, W.B., 2013. Systematic analyses of Ophiocordyceps lanpingensis sp. nov., a new species of Ophiocordyceps in China. Microbiol. Res., 168: 525-532. https://doi.org/10.1016/j. micres.2013.02.010

Driver, F., Milner, R.J. and Trueman, J.W.H., 2000. A taxonomic revision of Metarhizium based on a phylogenetic analysis of rDNA sequence data. Mycol. Res., 104: 134-150. https://doi.org/10.1017/ S0953756299001756

Kepler, R.M., Sung, G.H., Ban, S., Nakagiri, A., Chen, M.J., Huang, B., Li, Z.Z. and Spatafora, J.W., 2012. New teleomorph combinations in the entomopathogenic genus Metacordyceps. Mycologia, 104: 182-197. https://doi. org/10.3852/11-070

Kepler, R.M., Humber, R.A., Bischoff, J.F. and Rehner, S.A., 2014. Clarification of generic and species boundaries for Metarhizium and related fungi through multigene phylogenetics. Mycologia, 106: 811-829. https://doi.org/10.3852/13-319

Keyser, C.A., Licht, H.H.D.F., Steinwender, B.M. and Meyling, N.V., 2015. Diversity within the entomopathogenic fungal species Metarhizium flavoviride associated with agricultural crops in Denmark. BMC Microbiol., 15: 249. https://doi. org/10.1186/s12866-015-0589-Z

Kobayasi, Y. and Shimizu, D., 1978. Cordyceps species from Japan. Bull. natl. Sci. Mus., Tokyo Ser. B, 4: 43-63.

Larkin, M.A., Blackshields, G., Brown, N.P., Chenna, R., McGettigan, P.A., McWilliam, H., Valentin, F., Wallace, I.M., Wilm, A., Lopez, R., Thompson, J.D., Gibson, T.J. and Higgins, D.G., 2007. Clustal $\mathrm{W}$ and clustal $\mathrm{X}$ version 2.0. Bioinformatics, 23:
2947-2948. https://doi.org/10.1093/bioinformatics/ btm 404

Liang, Z.Q., Liu, A.Y. and Liu, J.L., 1991. A new species of the genus Cordyceps and its Metarhizium anamorph. Acta Mycol. Sin., 10: 257-262.

Nonaka, K., ōmura, S., Masuma, R., Kaifuchi, S. and Masuma, R., 2013. Three new Pochonia taxa (Clavicipitaceae) from soils in Japan. Mycologia, 105: 1202-1218. https://doi.org/10.3852/12-132

Pattemore, J.A., Hane, J.K., Wiliams, A.H., Wilson, B.A., Stodart, B.J. and Ash, G.J., 2014. The genome sequence of the biocontrol fungus Metarhizium anisopliae and comparative genomics of Metarhizium species. BMC Genom., 15: 660. https://doi.org/10.1186/1471-2164-15-660

Sanjuan, T., Tabima, J., Restrepo, S., Læssøe, T., Spatafora, J.W. and Franco-Molano, A.E., 2014. Entomopathogens of Amazonian stick insects and locusts are members of the Beauveria species complex (Cordyceps sensu stricto). Mycologia, 106: $260-275$. https://doi.org/10.3852/13-020

Shoukat, R.F., Freed, S. and Ahmad, K.W., 2018. Assessment of binary mixtures of entomopathogenic fungi and chemical insecticides on biological parameters of Culex pipiens (Diptera: Culicidae) under laboratory and field conditions. Pakistan J. Zool., 50: 299-309. https://doi:org/ 10.17582/ journal.pjz/2018.50.1.299.309

Sung, G.H., Hywel-Jones, N.L., Sung, J.M., Luangsaard, J.J., Shrestha, B. and Spatafora, J.W., 2007. Phylogenetic classification of Cordyceps and the clavicipitaceous fungi. Stud. Mycol., 57: 5-59. https://doi.org/10.3114/sim.2007.57.01

Tamura, K., Stecher, G., Peterson, D., Filipski, A., Nei, M. and Kumar, S., 2013. MEGA6: Molecular evolutionary genetics analysis version 6.0. Mol. Biol. Evolut., 30: 2725-2729. https://doi. org $/ 10.1093 / \mathrm{molbev} / \mathrm{mst} 197$

Wang, Y.B., Yu, H., Dai, Y.D., Chen, Z.H., Zen, W.B., Yuan, F. and Liang, Z.Q., 2015. Polycephalomyces yunnanensis (Hypocreales), a new species of Polycephalomyces parasitizing Ophiocordyceps nutans and stink bugs (hemipteran adults). Phytotaxa, 208: 34-44. https://doi.org/10.11646/ phytotaxa.208.1.3

Wen, T.C., Zha, L.S., Xiao, Y.P., Wang, Q., Kang, J.C. and Hyde, K.D., 2015. Metacordyceps shibinensis sp. nov. from larvae of Lepidoptera in Guizhou Province, southwest China. Phytotaxa, 226: 51-62. https://doi.org/10.11646/phytotaxa.226.1.5 